\title{
Designing Airport Interiors with 3D Visualizations
}

\section{Genovefa Kefalidou}

Department of Informatics

University of Leicester, LE1 7RH,

Leicester, UK

gk169@leicester.ac.uk

\section{André Castro}

Alma Design, Rua Armando Cortez

Ed. Interface, $n^{\circ} 1 C$ R/C D Paço de Arcos,

2770-233, Lisbon, Portugal

andre.castro@almadesign.pt

\author{
Mirabelle D'Cruz \\ Faculty of Engineering \\ University of Nottingham, NG7 2RD, \\ Nottingham, UK \\ Mirabelle.dcuz@nottingham.ac.uk
}

\section{Rui Marcelino}

Alma Design, Rua Armando Cortez

Ed. Interface, $n^{\circ} 1 C$ R/C D Paço de Arcos,

2770-233, Lisbon, Portugal

rui.marcelino@almadesign.pt

\section{ABSTRACT}

Understandings of user-centered design incorporate the need to include users and stakeholders in the design process from early on, employing visual and 'enactment' principles and approaches. Virtual Reality (VR) and 3D visualizations offer such opportunities for enhanced 'enactments' of proposed designs through immersion. Within PASSME H2020 European project, 3D design visualizations for novel concepts for an airport interior were developed and tested early on with users to identify the best interior design principles among the alternatives considered to reduce passenger stress, waiting times and improve overall Passenger Experience (PAX) ${ }^{1}$.
\end{abstract}

*Produces the permission block, and copyright information. See the specific order to use the table cells to include the authors in the order you want yourself and your co-authors to be listed.

Permission to make digital or hard copies of part or all of this work for personal or classroom use is granted without fee provided that copies are not made or distributed for profit or commercial advantage and that copies bear this notice and the full citation on the first page. Copyrights for third-party components of this work must be honored. For all other uses, contact the owner/author(s).

CHI'19 Extended Abstracts, May 4-9, 2019, Glagsgow, Scotland, UK.

(C) 2019 Copyright is held by the author/owner(s).

ACM ISBN 978-1-4503-5971-9/19/05.

DOI: https://doi.org/10.1145/3290607.XXXXXXX 


\section{Case Study Contributions:}

Advocating the value the VR and 3D visualization technologies can bring in usercentered design approaches for the interior design of complex mobility hubs such as airports

Demonstrating the successful elicitation of requirements and evaluation of proposed airport interior design by passengers themselves

Contribute to establishing guidelines for human-centered interior design developments

PASSME: The research presented in this paper was conducted as part of a Horizon2020 EU-funded project called PASSME (Personalised Airport Systems for Seamless Mobility and Experience) [12].
Using the potential of VR, concepts were tested with users and we identified passenger emotional and design-driven responses to boarding gates and lounge visualizations to inform the iterative development of in-situ passenger-centric interventions. We elicited both emotional, practical and operational needs and requirements for improving PAX within airports and found out that users can use VR to imagine interaction scenarios within the proposed design products.

\section{CCS CONCEPTS}

- Human-centered computing $\rightarrow$ Human Computer Interaction (HCI) $\rightarrow$ Interaction

Paradigms; Virtual reality

- Human-centered computing $\rightarrow$ Interaction Design; Empirical studies in interaction design

- Human-centered computing $\rightarrow$ Human Computer Interaction (HCI) $\rightarrow$ HCI Design and

Evaluation Methods; User Studies

- Human-centered computing $\rightarrow$ Visualization; Visualization design and evaluation methods;

- Human-centered computing $\rightarrow$ Interaction Design $\rightarrow$ Interaction Design Process and

Methods; User centered design

\section{KEYWORDS}

3D visualizations; airport interiors; passenger experience; emotional immersive design

\section{ACM Reference format:}

Genovefa Kefalidou, Mirabelle D'Cruz, André Castro, Rui Marcelino. 2019. Designing Airport Interiors with 3D Visualizations. In CHI Conference on Human Factors in Computing Systems Extended Abstracts (CHI'19 Extended Abstracts), May 4-9, 2019, Glagsow, Scotland, UK. ACM, New York, NY, USA. 4 pages. https://doi.org/10.1145/3290607.XXXXXXX

\section{INTRODUCTION}

The value of utilizing immersive technologies such as Virtual Reality (VR) and 3D visualizations in the design and evaluation process has increased in recent years $[6,2]$. This is usually because they allow to understand human behavior, interactions and experiences when engaging with such environments and they elicit information regarding any differences in interaction styles between them. By 'simulating' characteristics of the product prototype and visualizing it in such a manner, users are able to immerse not only within the product features but also get a further understanding of the potential in-situ context [6]. The main reason behind this is mainly due to the fact that such technologies have been found to support additional evaluation and feedback mechanisms (e.g. in regards to a designed product or prototype) that provide insights related to behavioral, physiological and cognitive interactions and characteristics [11]. There is existing research focusing on the utilization of VR and 3D visualizations in domains of training, learning and navigating e.g. [9]. However, limited studies have been arguing the value of VR and 3D visualizations as media for supporting design and usercentered design focusing mainly on either collaborative tasks (e.g. [8, 2]), manufacturing (e.g. [3]) or 


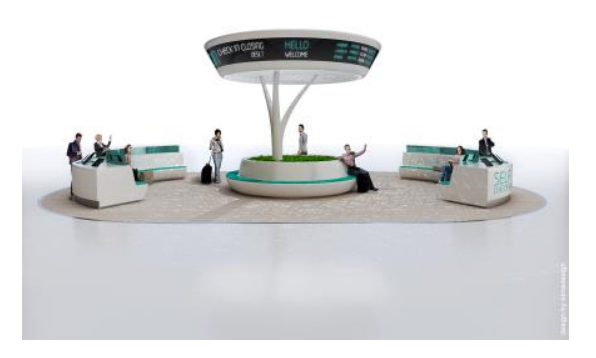

Figure 1: Design concept visualization for Check-in area.

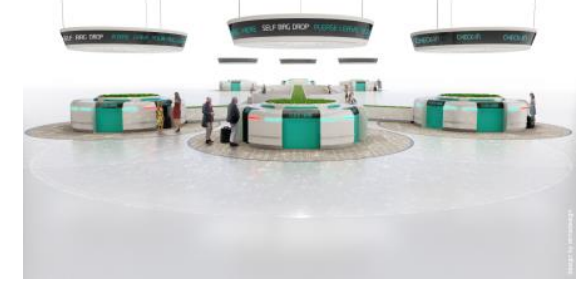

Figure 2: Design concept visualization for Luggage handling area.

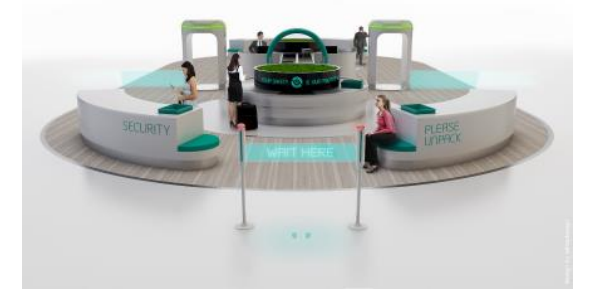

Figure 3: Design concept visualization for Security area. hospital interior design (e.g. [4]). More recently, research in 3D visualizations to support airport security operations and gates (e.g [7]) has emerged focusing on simulations. Considering the limited research in using $3 \mathrm{D}$ visualizations to support user-centered design for airport interiors to enhance passenger experience (PAX) and UX, the present research focuses on airport interior design, acknowledging the challenges, complexities and opportunities for enhancing UX and PAX for a seamless journey. Recent surveys (e.g. [5, 1]) suggest that passengers exhibit a wide range of different emotions when at airport, depending on what stage (i.e. check-point) they are within their of their airport journey. Indeed, PAX at airports appears to be a sum of different factors, which include both the physical environment - i.e. the look and feel of the premises - as well as the processes and encounters between people. In PASSME [10] we focused on enhancing passengers experience at airports, through optimizing interiors, luggage flow and offering real-time personalized information. In the remaining sections of the paper, we are discussing the design process adopted within our present research and highlight key findings.

\section{THE DESIGN PROCESS METHOD}

As mentioned earlier, the purpose of these design- focused studies was to identify user and passenger (as our participants were both users of our 3D visualizations and airport passengers) impressions, needs and evaluations of proposed airport interior visualizations through the utilization of immersive technologies such as VR. The proposed visualizations aimed to improve PAX when passengers reside or go through airport complexes such as security areas, lounges, gates and check-in. The main objective was to capture user feedback when exposed to a series of airport interior visualization prototypes designed by Alma design. The prototypes were developed within PASSME project and used different design principles as guidelines for the interior design of four specific airport areas / touchpoints: welcome area and check-in; baggage drop off; security; boarding gates.

The design of the prototypes started by the layout of the areas, trying to provide passengers with unobstructed views of the information displays, shaped in elevated circular displays. The circular layouts were also found to potentially promote social interaction between users and improve circulation. The concepts were developed around certain design guidelines, such as the use of round, soft shaped furniture, neutral colors and reference to natural elements to promote the biophilia effect [4] - where environments rich in nature view and imagery are proved to reduce stress and enhance focus and concentration. With the objective of optimizing processes and enhancing PAX, the welcome area featured a circular layout seating area, curved furniture and self-check-in devices. This area worked as a meeting point, promoting sociability between passengers (Fig. 1). The luggage handling area (drop-off) featured circular islands with drop-off hardware and user interface for passengers. This layout was designed to promote informal queuing, easy flow of passengers and featured a large central display of information (Fig. 2). The Security area is based on a layout where curved furniture (for tray retrieval) and circular furniture (featuring nature elements) help distribute passengers around the unpacking areas. The Boarding Gates area featured a semi-circular layout in which passengers could 


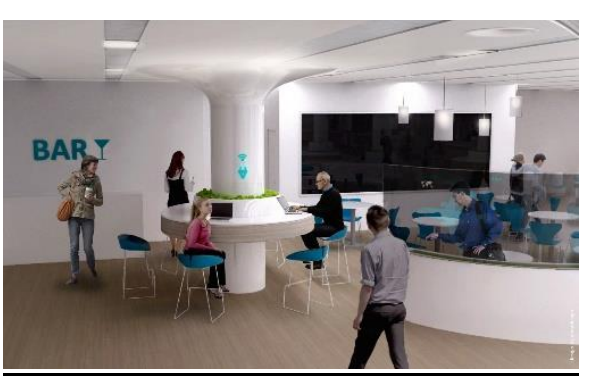

Figure 4: Boarding Gates Visualization (waiting area near bar).

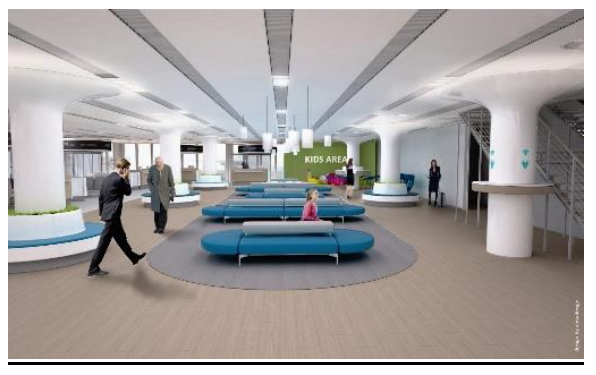

Figure 5: Boarding Gates Visualization (waiting area near the kids' area).

Participants: Twelve individuals took part in this evaluation study $\left(M_{\mathrm{age}}=32\right.$ years old; 5 Male, 7 Female; Age range: 23-62; Background: academic, nonacademic, admin and students; All experienced flyers ( $>5$ years). All participants were reimbursed with $£ 10$ Amazon vouchers. Ethics approval was gained through the University of Nottingham, Department of Engineering, Ethics Committee. sit, work, relax and check for flight information in a large circular display placed at eye-level (Fig. 3). The design concepts were developed using different design methodologies, such as sketching sessions, mood-boards, concept selection session, concept refinement, 3D CAD modeling and rendering. A coherent design language was used throughout the four different areas, combining an expression of round shapes, the use of bright, white finishes and colorful details for affordance. The final visualizations developed covered all the key PASSME touch points for airport interiors e.g. check-in and luggage handling (drop-off), security and boarding gate.

\section{THE STUDY}

In the case of the Boarding Gate areas (Fig. 4 and 5), a new general layout of the area was proposed. Furthermore, seat layouts for different activities we designed and new boarding counters and information displays. The seat arrangement aimed to follow a "fluid" layout and to create different areas for different activities: rest, relax, work, talk, play (taking into account group and family travel), etc. maintaining clear visibility lines throughout the gate (in order for the passengers to clearly see their boarding counter / gate). A round pillar concept was developed to create social areas and include work / recharge areas with high seats / tables. The pillar allowed for different configurations: Social area (with wrap-around seats and flower-box); Working area (with wrap-around working tables and high seats); Connectivity area (where each pillar provides charging points and wired connectivity). Differentiated floor aimed to separate boarding areas from the lounge areas and a specific "kids" area for children was developed as well as a "bar" area. All proposals were developed around certain design guidelines, such as the use of round, soft shaped furniture (soft corners) bright colors and reference to natural elements to promote the biophilia effect. Natural materials and finishes were applied to create a comfortable, cozy environment. The main objectives were to capture user feedback when exposed to a second round of airport interior (3D this time) visualization prototypes designed by Alma Design (based on the Bus Gates area at the Hamburg (HAM) airport) and to utilize $360^{\circ}$ google cardboard prototypes for participants to view the visualizations in order to identify potential changes in how participants experience interior designs through VR technologies.

\subsection{Procedure}

In six interactive sessions (of 2 people each) participants had to use the $360^{\circ}$ google cardboard prototypes (both at the same time within each session) to view the visualizations and comment upon them through questionnaires and open discussion. They were informed about the study and VR usage (including risks). Once consented, participants used Google Cardboards to view the same $360^{\circ}$ airport interior visualizations in each phase (see Fig. 4 and 5 for examples). During viewing, participants discussed their experience, feelings and impressions of the airport interiors amongst themselves. Once finished with viewing, participants completed a questionnaire that included a number of 7-point subjective attitude scales (ranging from: 'unhappy'-'happy'; 'calm'-'excited'; 'controlled'-'incontrol'; 'unpleasant'-'pleasant'; 'negative'-'positive') regarding demographics, the visualizations, comfort and incentives for use. Sessions were audio-recorded. 


\section{FINDINGS AND DISCUSSION}

Table 1: Participant Responses on

Boarding Gate area visualization (Fig. 4) emotion responses and perceptions of aesthetics

\begin{tabular}{llll}
\hline $\begin{array}{l}\text { Unhappy/ } \\
\text { Happy }\end{array}$ & Calm/ & $\begin{array}{l}\text { Controlled/ } \\
\text { Excited }\end{array}$ & $\begin{array}{l}\text { Negative/Positive } \\
\text { Introl }\end{array}$ \\
\hline 4.00 & 3.83 & 4.00 & 4.25 \\
\hline
\end{tabular}

Table 2: Participant Responses on Boarding Gate area visualization (Fig. 4) environment adjectives/characteristics and confusion levels

\begin{tabular}{ll}
\hline $\begin{array}{l}\text { Environment } \\
\text { characteristics } \\
\text { (frequencies) }\end{array}$ & $\begin{array}{l}\text { Confusion in the scene } \\
\text { (Disagree/Agree) }\end{array}$ \\
\hline $\begin{array}{l}\text { Futuristic (4) } \\
\text { Minimal (8) }\end{array}$ & 4.92 \\
\hline
\end{tabular}

Table 3: Participant Responses on

Boarding Gate area visualization (Fig. 5) -

emotion responses and perceptions of

aesthetics

\begin{tabular}{llll}
\hline $\begin{array}{l}\text { Unhappy/ } \\
\text { Happy }\end{array}$ & $\begin{array}{l}\text { Calm/ } \\
\text { Excited }\end{array}$ & $\begin{array}{l}\text { Controlled/ } \\
\text { In-Control }\end{array}$ & $\begin{array}{l}\text { Negative/Positive } \\
\text { Aesthetics }\end{array}$ \\
\hline 4.75 & 3.83 & 4.83 & 4.50 \\
\hline
\end{tabular}

\subsection{Negative Emotional Responses}

The results are being presented below under the themes emotional responses (negative, positive, neutral) as well as emerged needs following a light thematic analysis approach. Tables 1 and 2 show the mean responses as given by participants when exposed to 'boarding area 1' (Fig. 4) and Tables 3 and 4 show the mean responses for 'boarding area 2' (Fig. 5) 3D (i.e. 360o). The waiting boarding area close to the bar exhibited less control, lower happiness levels and aesthetics and more confusion as to that they were expected to do in a space as represented in the image (Fig. 4). While participants were able to understand that this visualization represented a seating area, it was not clear to them what this seating area was for, emphasizing the need for interior design cues to understand the surrounding space. The waiting boarding area close to kids' area, on the other hand, triggered more happiness, higher levels of control and lower levels of confusion as its purpose seemed evident to participants.

\subsection{Positive Emotional Responses}

It appeared that the visualization of the waiting boarding area close to the kids' area (Fig. 5) inspired more control and happiness to participants. An explanation provided by participants for this response was that the kids' area can provide notes of relaxation just before boarding while at the same time it can potentially relax both parents and kids as the latter are playing while parents can occupy themselves otherwise as well. Participants acknowledged that 3D visualizations can potentially be very important artefacts and ways to 'experience' a space as they allow more dimensions in movement. Participant 10 (P10) particularly said that for interior design solutions it is important to be able to immerse into the space as it somehow changes the way things are imagined (See 'Participant Quotes'). It seems that the use of 3D visualizations could have a potential to open up new 'cases' and 'scenarios' for users and designers to consider within a participatory and user-centered design setting. Furthermore, participants selected more words to describe the interior design representing the waiting boarding area near the kids' area (Fig. 5) (Table 4) by reporting that it felt minimal, futuristic but also traditional and natural. They also rated Fig. 5 (i.e. waiting boarding area close to the bar) as being minimal and futuristic only. It seems that for the visualization that they felt happiest and more relaxed with (i.e. Fig. 4), familiarity, potential for utility (i.e. relaxation for both parents and kids) alongside with minimalism and futurism (i.e. something being 'of the future') appear to be important factors for responding positively to an interior design approach (e.g. see 'Participant quotes').

\subsection{Neutral Emotional Responses}

As seen on Tables 1 and 2, participants overall reported mostly average responses (around $\mathrm{M}=4.00$ ) in regards to the emotions that the waiting area (i.e. boarding area) visualizations exhibited to them. 
Table 4: Participant Responses on Boarding Gate area visualization (Fig. 5) environment adjectives/characteristics and confusion levels

\begin{tabular}{ll}
\hline $\begin{array}{l}\text { Environment } \\
\text { characteristics } \\
\text { (frequencies) }\end{array}$ & $\begin{array}{l}\text { Confusion in the scene } \\
\text { (Disagree/Agree) }\end{array}$ \\
\hline $\begin{array}{l}\text { Futuristic (5) } \\
\text { Natural (1) }\end{array}$ & 3.41 \\
Traditional (1) & \\
Minimal (5) & \\
\hline
\end{tabular}

\section{Participant Quotes:}

P1: "It is fun looking at these [3D visualizations], it would be nice to be a bit more interactive with the stuff in there"

P3: "“'It is nice to see space for kids, in that, I mean, it is easy to get them occupied with something different other than smartphones (...) not sure how noisy that would be and disruptive for others (...)"

P6: "It is nice but annoying also because you look up and you see nothing (...) it would be nice if it was a more complete model, I would like to see and experience that"

P10: "Seeing it through google cardboard makes me feel nice, well provided I do not get dizzy or so, it makes me think of what would happen if I go over there"
Participant 3 acknowledged both positive and challenging points of this design proposal (Fig. 5) highlighting that it would potentially be good for parents and children but not necessarily enjoyable by other passengers that are solo or need to work, highlighting the social context of the surroundings.

\subsection{Emerged Needs}

In regards to the use of $3 \mathrm{D}$, participants emphasized the potential value of such technologies to support user-centered and agile design processes but also discussed about the 'playfulness' it promotes within the process as Participant 1(P1) suggests (see 'Participant Quotes') . Participant 6 (P6) pointed out as well that because the experience in 3D is indeed immersive, it would be beneficial to test designs with different 'realism' levels - it somehow creates an 'anticipation' and 'want more' experience (see 'Participant Quotes'). According to participants, signage within the visualized proposed designs are important, however, they highlighted the use of alternatives (e.g. mobile applications and smartphones) as providing opportunities for de-cluttering the physical informational space within airports. For example, a way to avoid multiple co-located signage (that may confuse passengers) would be to provide personalized travel information (e.g. personal wayfinding and pathway to airport touch points) on the mobile screen and as needed by each passenger individually. This, by no means, suggests that signs should be dismissed from the airports, instead, indoor airport signage should focus on the most important landmark information signage avoiding confusion by having duplicate (and sometimes conflicting) signage. Participants also suggested that information needs within such indoor spaces (e.g. waiting areas and boarding gates) may indeed vary depending on the mode of travel and the type of copassengers. For example, if traveling with a group of people that may include vulnerable individuals such as children or older individuals, information about time becomes even more mandatory so that any information as to how much time is left to board (or to need to go from point A to point B) is appreciated. So, within the context of the present 3D visualizations, information points (or stands) embedded within the indoor design could be useful for creating an atmosphere of constant awareness, realism and safety. Information provision, indeed, at any point while at airports, appears to be a fundamental feature of airport interior design. Participants who viewed the visualizations above expressed the need to have visually displayed information about their flight status and their needs alongside the proposed interior design elements. For example, in both Fig. 4 and Fig. 5, participants noticed the lack of any information provided apart from the signs indicating 'kids' area' and 'bar'. Furthermore, even such signs were not considered enough to inspire them certainty as to where in their travel flow are (e.g. in terms of time left, in terms of whether they are at the right gate and in terms of where they can find additional facilities).

\subsection{Discussion}

Amongst our findings, it was identified that passengers required intuitive cues embedded in the airport design which pointed towards what services were available and information about their journey. In particular, they appreciated spaces that facilitated fun and relaxation, such as a children's play area whereby passengers can de-stress. At the same time, however, they emphasized the need to receive personalized information about their travels (and their next steps) in such a way that they do not get 


\section{ACKNOWLEDGMENTS}

This research was conducted under PASSME. PASSME has received funding from the European Union's Horizon 2020 research and innovation programme under grant agreement No 636308'. 'The opinions expressed in this document reflect only the authors' view and reflects in no way the EuropeanCommission's opinions. The European Commission is not responsible for any use that may be made of the information it contains.

Many thanks are also due to all our participants and to Neil de Joux, Vicki Antrobus and Davide Salantiri for all their help and support in data collection.

\section{REFERENCES}

[1] ACI EUROPE. 2014. Guidelines for Passenger Services at European Airports.

2] S. Aromaa and K. Vaananen. 2016. Suitability of Virtual Prototypes to support Human Factors/Ergonomics Evaluation during the Design. Applied Ergonomics. 56 (2016), 1118.

[3] C.J. Chen. 2000. Virtual Reality in Interior Design: A Prototype System. Virtual Reality: Select Issues and Applications. 63-78.

[4] P.S. Dunston, L.L. Arns, J.D. Mcglothlin, G.C. Lasker and A.G. Kushner. 2011. An Immersive Virtual Reality Mock-up for Design Review of Hospital Patient Rooms. Collaborative Design in Virtual Environments, 167-176.

[5] B. Grinde and G. Patil. 2009. Biophilia: Does Visual Contact with Nature impact on Health and Well-being? International Journal of Environmental Research and Public Health. 6, 9 (2009), 2332-2343. confused or overloaded with information. Participants have acknowledged the potential value of utilizing VR technologies as a means for travel planning both when at airport and also prior to arriving at airports similarly to what [7], [9] and [4] suggest. For example, such 3D visualizations, can prepare passengers, who have not arrived at the airport yet, about the surroundings and navigation aspects of the departure airport. In that way, situation awareness efforts would be minimized in regards to both issues of walkthrough and presence of available facilities. At the same time, the utilization of such visualizations within the design and evaluation process allowed participants to not only experience traditional design stages such as inspiration, generation and embodiment and identify potential usability issues but also were able to infer and design additional user scenarios and personas through their immersion in the 3D space as [7] also posit.

Indeed, that immersion elicited additional feelings of 'anticipation' and 'want-more' interactivity acknowledging the importance of having more complete and fully interactive 3D models to 'play with'. This latter point was indeed one of the limitations of our study (as the 3D visualizations did not incorporate full 3D interactive models) as the aim of the study was to elicit first impressions from users on airport interiors rather than provide a platform of 'role-playing' scenarios within that context indeed, this could be a clear direction for future research in incorporating VR in user-centered design processes. Our participants showed self-reflection instances when interacting with the 3D airport interior visualizations that open up opportunities to re-consider the value that we -as HCI community - gain through the use of such immersive technologies. Furthermore, considering VR technologies as self-reflection tools can create new conceptual and applied frameworks for user-centered design and systems' evaluation alongside with more traditional methods of qualitative and quantitative methods. One of the reasons for having appropriate 3D models of airport interiors built in is so that they can provide sufficient levels of realism and movement within the suggested airport interior design. In particular, People with Reduced Mobility (PRMs) and other vulnerable passengers (e.g. older individuals and children) could potentially get value from such interventions for both planning and entertainment purposes. Through the process of utilizing $3 \mathrm{D}$ visualizations for airport interior design, we managed to elicit both design, navigational, interactional and information needs that passengers may have if they were to be immersed and exposed to the proposed interior designs. For example, the presence of cues was highlighted as important for navigational purposes but also the importance of balancing out both aesthetic and utility principles to enhance PAX and interactivity with the space.

Additionally, visualizations helped participants consider aspects of 'realism', especially in response to volumes of passengers and how they would 'behave' within the proposed airport interior designs. This has emphasized greatly the importance of 'realism' in generating interior design prototypes and highlighted the possibilities that VR can offer in this direction. For example, 3D models can be built based on different 'volume' scenarios where different numbers of characters are embedded in and provide a realistic (not real) perception and simulation of space. Indeed, our participants discussed about 'properties' of space and how these include both time, volumes of passengers, presence of cues, interior furniture and information provision. Furthermore, our participants emphasized that VR technologies provide affordances for 'plug and play' design features, especially for testing options for 


\section{REFERENCES (Continued)}

[6] J. Jerald. 2015. The VR Book: Humancentered Design for Virtual Reality. Morgan \& Claypool.

[7] G. Kefalidou, M. D’Cruz,... and E. Shaw. 2016. Passengers' Requirements for developing a Passenger-centred Infrastructure to enhance Passenger Experiences at Airports. In Proceeding of the Chartered Institute of Ergonomics and Human Factors Conference (CIEHF 2016).

[8] D.B. Koch. 2004. 3D Visualization to support Airport Security Operations. IEEE Aerospace and Electronic Systems Magazine. 19, 6 (2004), 23-28.

[9] P.Koutsabasis, S. Vosinakis, K. Malisova and N. Paparounas. 2012. On the Value of Virtual Worlds for Collaborative Design. Design Studies. 33, 4 (2012), 357-390.

[10] S. Lee. 2009. The Development of 3D Visualization Technology: The Potential Impact on Interior Design and its Consumers. International Journal of Consumer Studies. 33, 5 (2009), 611-617.

[11] V. Rieuf, C. Bouchard, V. Meyrueis and J-F. Omhover. 2017. Emotional Activity in Early Immersive Design: Sketches and Moodboards in Virtual Reality. Design Studies. 48, 5 (2017), 43-75.

[12] PASSME Website. 2015. Retrieved December 172018 from https://passme.eu/ 'cluttering' and 'decluttering' interior spaces. Finally, in regards to PAX and UX, VR can provide different layers of personalization during the design phase where users can 'build up' scenarios personas (such as for PRMs and different modes of travel) to evaluate more and successfully and more 'realistically' proposed designs.

\section{CONCLUSIONS AND NEXT STEPS}

Results from this study demonstrated the need for interior design cues to understand the surrounding space and expressed the need for the presence of both aesthetic and utility principles when designing airport interiors. Furthermore, a balance between familiarity and novelty (or futurism) can provide the appropriate levels of impetus and exciting in using airport interiors and perceiving them positively. Decluttering the physical informational space within airports is important and modern mobile technologies (and Virtual Reality - VR) can offer opportunities for providing such realizations. A key challenge that emerges within airport interior design is how to provide affordances for different passenger groups (e.g. PRMs, group travel etc.) without compromising space aesthetics and utility while support informational needs without creating additional cognitive or emotional demand to the passenger. A next step would be to start building up a set of guidelines for incorporating (more often) 3D visualizations and VR/AR within the design process and viewed both as design products but also as design tools that facilitate immersion and opportunities for new 'preliminary' interactions and generation of 'immersive scenarios' in a given context. As such, we posit that -especially in interior design- design processes should incorporate 3D prototypes to generate complementary impressions for a proposed product or space and track mood and emotional responses in both cases. This can be particularly important for complex 'mobility hub' interior spaces such as airports, train and bus stations where passengers engage in a number of different tasks that can influence their travel experience in multiple levels. The incorporation of tactile feedback would also be advantageous in providing an additional level of experience to the users as and when they immerse in the 3D visualizations. 